\title{
DISRUPTIVE BEHAVIOUR AMONGST DOCTORS, MYTH OR REALITY?
}

Avtar Singh Bansal ${ }^{1}$

\section{HOW TO CITE THIS ARTICLE:}

Avtar Singh Bansal.“Disruptive Behavior amongst Doctors, Myth or Reality?”Journal of Evolution of Medical and Dental Sciences 2014; Vol. 3, Issue 02, January 13; Page: 399-406, DOI:10.14260/jemds/2014/1841

ABSTRACT: BACKGROUND: Disruptive behavior in a medical setting is defined as objectionable or offensive interpersonal behavior that leads to disruption of professional activities in the workplace. ${ }^{1}$ It has been observed that majority of doctors do not show disruptive behavior in their day today conduct and only few doctors are identified for their disruptive behavior. Special committee on professional conduct and ethics defines disruptive behavior in physicians as aberrant behavior manifested through personal interaction with physicians, hospital personnel, health care professionals, patients, family members or others which interferes with patient care or could reasonably be expected to interfere with the process of delivering quality care. ${ }^{2}$ Common forms of disruptive behaviors generally seen amongst young doctors are use of abusive language, yelling or shouting at patients, colleagues and subordinate staff, showing indisciplined behavior and at times indulging in physical abuse. ${ }^{3-4}$ STUDY DESIGN: Study was conducted at a tertiary care hospital where 614 health care professionals participated which included 108 doctors 432 nursing staff and 74 paramedical staff METHOD: Data collection was done by semi structured pretested questionnaire and was entered in Microsoft Excel and analyzed for frequency and percentages. RESULTS: $64 \%$ doctor, $66 \%$ nursing staff and $50 \%$ of the paramedicals answered that they have seen doctors showing disruptive behavior at one time or the other. Not all the doctors show disruptive behavior but this type of aberrant behavior is seen mainly in2-3 percent of doctors only. While answering to the question as to the type of disruptive behavior, 57\% health care professionals reported that commonest form of disruptive behavior noticed by them amongst doctors was yelling or shouting on junior staff, patients and colleagues. $47 \%$ answered that doctors with disruptive behavior do not follow laid down orders or protocols, $1 \%$ respondents reported that they have seen doctors showing caste discrimination amongst staff. Sexual remarks or physical abuse was reported by negligible number of respondents. CONCLUSION: Majority of health care professionals reported that problem of intimidating or disruptive behavior does exist in small minority of doctors and while working with such doctors it causes mental stress, frustration, inability to concentrate on work, fear to communicate, reduction in teamwork and relations with such doctors remain tense.

KEYWORDS: Disruptive behavior, Doctors, Health care professionals, Nursing staff, Communication, Behavioral and psychological responses, Adverse events.

INTRODUCTION: Incidences of disruptive behavior in health care settings are very common and many researches have documented the frequency and impact of disruptive behaviors of doctors on the staff relationships and clinical outcomes of cases ${ }^{5}$. Disruptive behavior is defined as any inappropriate behavior, confrontation, or conflict, ranging from verbal abuse (yelling, intimidation, condescending, berating, disrespectful, abusive behaviors) to physical or sexual harassment that can negatively impact work relationships, communication efficiency, information transfer and the process and outcomes of care ${ }^{6}$. It's not only that doctors are responsible for showing disruptive behavior in health care setups but nurses also show disruptive behavior with equal frequency, 
however the difference between the two is that doctor's disruptive behavior directly influences patient care. The disruptive behavior amongst doctors is overt phenomenon which quickly unfolds during the course of treatment and ends soon after whereas amongst the nursing staff this behaviour takes more of a passive/aggressive approach, with behind-the-scenes undermining and subterfuge and most of the activity is directed at other nurses. Some have applied the phrase "horizontal hostility" to describe its nature ${ }^{7}$. In the past, hospital administrators, nurses, employees and even patients and their families have often been tolerating the aberrant physician behavior. The management generally overlooks the disruptive behaviour by doctors who are high producers and attract large number of patients. It is advocated that this "high strung" behavior merely reflects high stress in an overworked doctor. Disruptive behaviour of doctors leads to stress, frustration and low morale of subordinate staff. Findings of studies have suggested that a relationship does exist between workplace stress and commitment of staff to the organization, job satisfaction and desire to quit the job.8-12 People are now becoming increasingly aware about the importance of team communication in the delivery of quality patient care. ${ }^{1}$ Disruptive behaviour may lead to adverse events, medical errors, compromises in patient safety and can affect patient mortality as well. ${ }^{13}$

MATERIALS AND METHODS: A 21 question survey tool was distributed to doctors, nurses and paramedical staff in a tertiary care hospital to assess their responses for occurrence of disruptive Behavior amongst doctors, its impact on staff behavior, communication efficiency and patient care. Questions were in the form of yes/no, multiple choice and 5 point ratings-based Likert scales. The survey was reviewed and tested for its validity. 108 doctors, 432 nursing staff and 74 technicians totalling 614 health care professionals participated in the survey.

RESULTS: First question asked to all the participants was whether they had seen disruptive behavior amongst the doctors at any time? 69 (63.89\%) doctors, 287 (66.44) nurses and 37 (50\%) paramedicals reported that they had witnessed disruptive behavior in doctor at some or the other time. The total percentage of response from all health care professionals $(n=614)$ who had witnessed the disruptive behavior amongst doctors was 393(64.01\%).(Table -1).

\begin{tabular}{|c|c|c|c|c|c|c|}
\hline Type of Staff & No. & $\begin{array}{c}\text { Witnessed } \\
\text { Disruptive } \\
\text { Behavior }\end{array}$ & $\begin{array}{c}\text { Daily } \\
\text { No/ } \\
\text { Percent }\end{array}$ & $\begin{array}{c}\text { Weekly. } \\
\text { No/ Percent }\end{array}$ & $\begin{array}{c}\text { Monthly. } \\
\text { No/ Percent }\end{array}$ & $\begin{array}{c}\text { Yearly. } \\
\text { No/ Percent }\end{array}$ \\
\hline Doctors & 108 & $69(63.89 \%)$ & $11(10.19 \%)$ & $32(29.63 \%)$ & $37(34.26 \%)$ & $28(25.93 \%)$ \\
\hline Nursing Staff & 432 & $287(66.44 \%)$ & $48(11.11 \%)$ & $88(20.37 \%)$ & $113(26.16 \%)$ & $183(42.36 \%)$ \\
\hline Paramedicals & 74 & $37(50 \%)$ & $11(14.86 \%)$ & $19(25.68 \%)$ & $28(37.84 \%)$ & $16(21.62 \%)$ \\
\hline Total & 614 & $393(64.01 \%)$ & $70(11.40 \%)$ & $139(22.64 \%)$ & $178(28.99 \%)$ & $227(36.97 \%)$ \\
\hline \multicolumn{7}{|c|}{ Table1: Frequency of disruptive behavior amongst doctors }
\end{tabular}

Next question was asked about the frequency of disruptive behavior seen in doctors. Whether it is daily, weekly, monthly or yearly.10.19\% doctors, $11.11 \%$ nurses and $14.86 \%$ paramedicals said that they have been witnessing it every day.29.63\% doctors, $20.37 \%$ nurses and $25.68 \%$ paramedicals seen it once a week. $34.26 \%$ doctors $26.16 \%$ nurses $37.84 \%$ paramedicals witnessed once a month and $25.93 \%$ Doctors, $42.36 \%$ Nurses and $21.62 \%$ only once or twice in a 
year. Overall Disruptive behavior amongst doctors was witnessed daily by $11.40 \%$ health care professionals, once a week by $22.64 \%$, once a month by $28.99 \%$ and once or twice in a yearby $36.97 \%$ respondents(Table 1 ).

When enquired about number of doctors showing disruptive behavior. 172 (28.01\%) respondents said that they have seen only one percent of doctors with aberrant or disruptive behavior, 191(31.11\%)persons answered that only 2-3 percent of doctors show disruptive behavior, $113(18.40 \%)$ reported disruptive behavior in 4-5\% of doctors, $79(12.87 \%)$ in6-10 percent of doctors and54(9.61\%)in 6-10 \% in more than ten percentage of doctors(Fig 1).

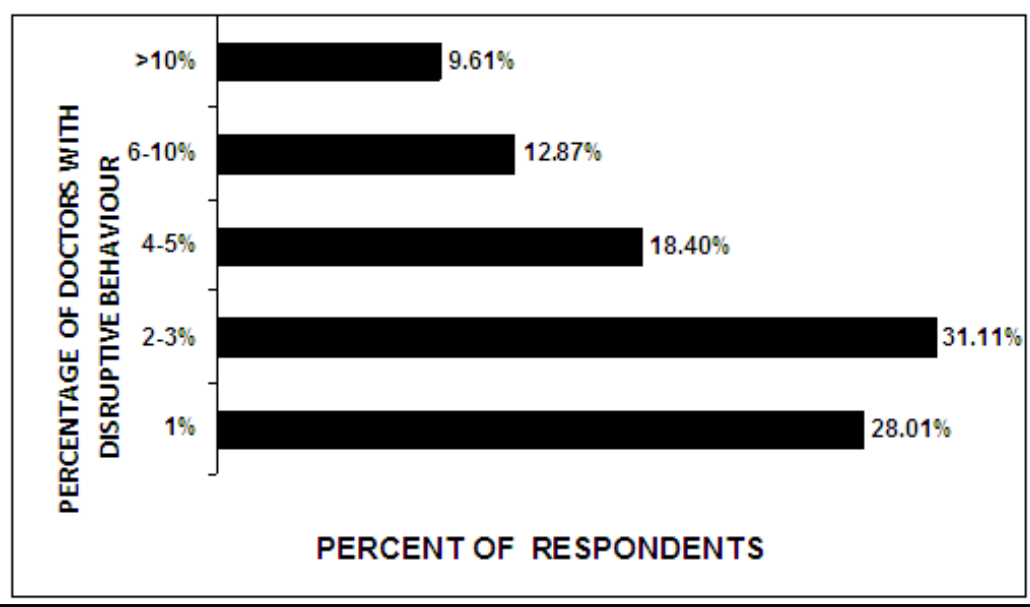

\section{Fig. 1: Percentage of doctors who exhibit disruptive behaviour}

Health care professionals were asked to identify the doctors that exhibit disruptive behavior. $396(64.5 \%)$ reported that it is same identified small number of doctors that are responsible for showing disruptive behavior time and again.

On seeking information about affect of gender on disruptive behavior, 68 (62.96\%) doctors, $242(56.02 \%)$ Nurses and 41 (55.41\%) paramedicals said that it is more amongst male doctors, whereas14(12.96\%) doctors, 79 (18.29\%) nurses and $15(20.27 \%)$ paramedicals were of the view that it is observed more amongst female doctors. 26 (24.07\%) doctors, 111(25.69\%) nursing staff, $18(24.32 \%)$ paramedicals reported no difference in the gender. Overall $57.17 \%$ health care professionals wrote that disruptive behavior is more amongst male doctors, $17.59 \%$ in female doctors and $25.24 \%$ respondents did not find any gender difference.

\begin{tabular}{|c|c|c|c|}
\hline Respondents & Male & Female & No difference \\
\hline Doctors & $68(62.96 \%)$ & $14(12.96 \%)$ & $26(24.07 \%)$ \\
\hline Nursing staff & $242(56.02 \%)$ & $79(18.29 \%)$ & $111(25.69 \%)$ \\
\hline Technicians & $41(55.41 \%)$ & $15(20.27 \%)$ & $18(24.32 \%)$ \\
\hline Total & $351(57.17 \%)$ & $108(17.59 \%)$ & $155(25.24 \%)$ \\
\hline
\end{tabular}

Table 2. Influence of gender on disruptive behavior

Figure 2 describes the specific types of disruptive behaviors and their frequency noted amongst doctors. $347(56.51 \%)$ respondents were of the opinion that the commonest type of disruptive behavior noted by the min doctors with disruptive behavior was shouting or yelling at 
staff. 287 (46.74\%) persons answered that these doctors do not follow laid down rules and protocols, 187 (30.46\%) reported disrespectful interaction with colleagues and staff, 84 (13.68\%) reported non cooperation, $75(12.21 \%)$ said that these doctors either do not respond to phone calls or if at all pick the phone they start shouting rather than rendering advice on patient care, $52(8.47 \%)$ wrote that they insult staff and colleagues in front others, 47 (7.65 \%) felt discriminated, $42(6.84 \%)$ witnessed throwing of instruments or article in a fit of anger and $17(2.77 \%)$ spoke ill of colleagues, 15 (2.44\%) wrote that doctors addicted to tobacco or alcohol. The aberrant behaviored doctors spoke minimally about cast or use of physical force.

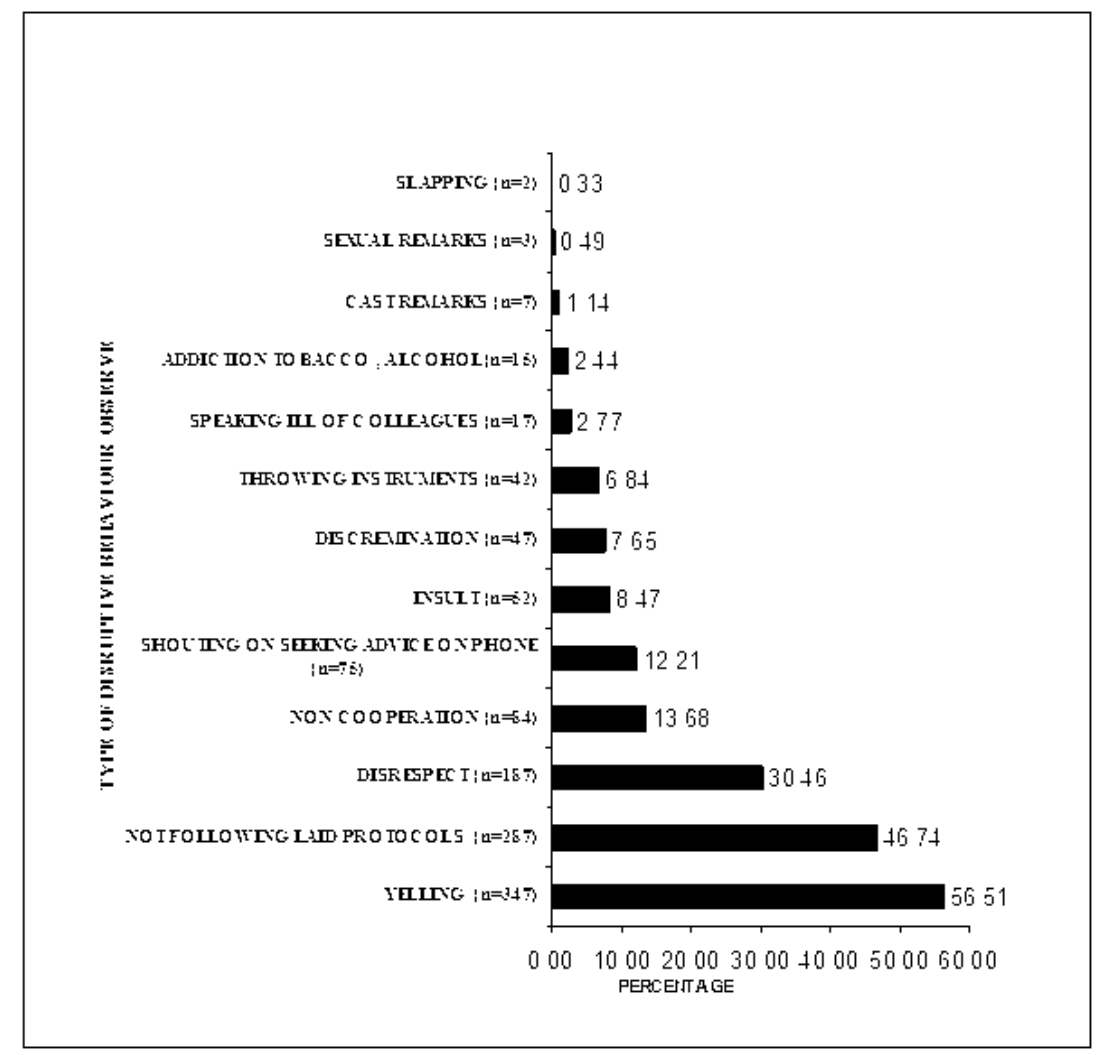

\section{Fig. 2: Frequency and type of disruptive behaviours amongst doctors}

In this study participating health care professionals were asked to write the effects of disruptive behavior on the behavioral and psychological responses of staff in terms of stress, frustration, loss of concentration, reduced communication and impairment of relation

Figure 3 summarizes the results for each of these factors. 513 (83.55\%) of the respondents said that they get mentally stressed by the disruptive behavior of the doctors, 469 (76.38\%)respondents wrote that it leads to frustration, $374(60.81 \%)$ were not able to concentrate while working, $262(42.67 \%)$ were scared to communicate with such doctors as these doctors may insult them, 313(50.98\%) respondents wrote that relation with such doctors become tense and impaired and 293 (47.72\%) replied that it leads to reduction in teamwork. 


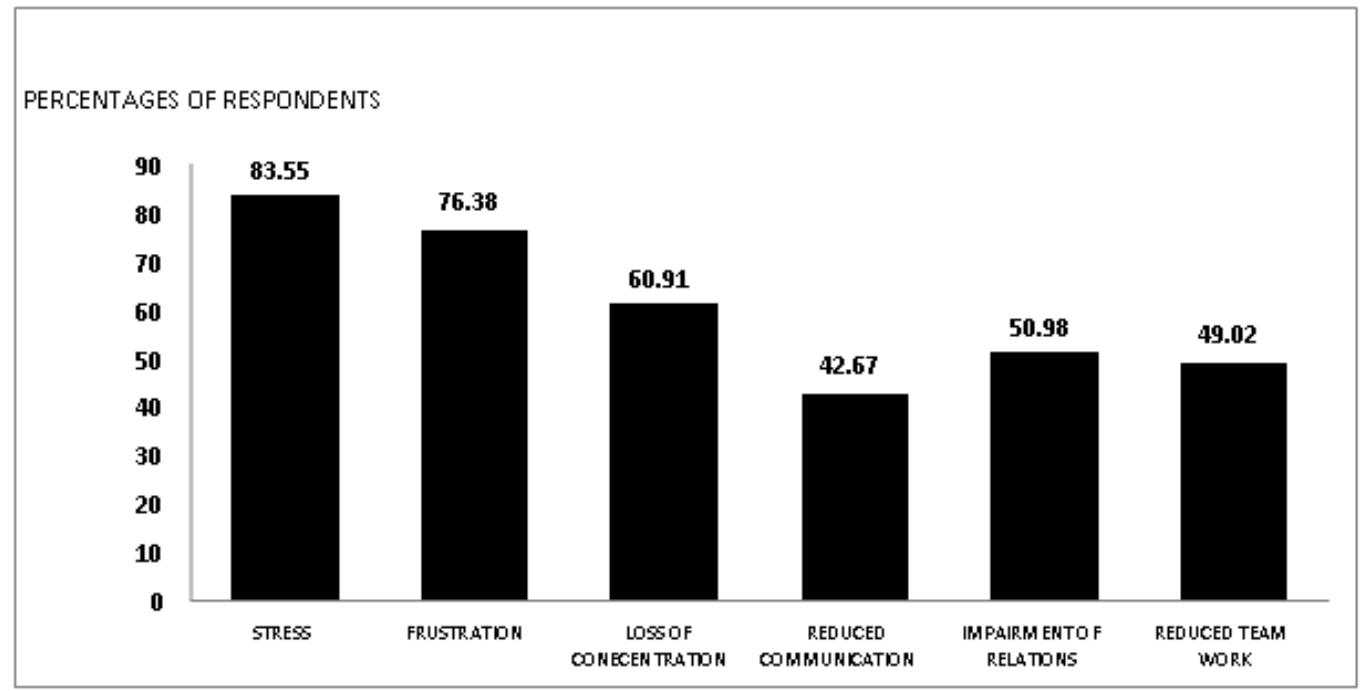

Fig. 3: Affect of disruptive behaviour of doctors on staff

Another question asked was that how serious an issue disruptive physician behavior was at the hospital. The overall response to this question was 3.34 on a scale of $1-5$, with 5 being very serious.

DISCUSSION: Intimidating and disruptive behaviours in health care organizations are not rare. ${ }^{14-17}$ Search of literature did not reveal any such study ever conducted in India. Many studies on this subject have been undertaken abroad and these studies bring out the fact that disruptive behaviour amongst doctors is an issue that requires to be addressed. The hospital management, other doctors, nurses, staff and even patients have been tolerating such aberrant behaviour of doctors. In fact, management in health care settings does not want to lose doctors with disruptive behaviour who are of high caliber and attract large number of patients. They simply tolerate aberrant behaviour of these doctors thinking that "high strung" behaviour is merely due to high stress in an overworked doctor.

In present study it was observed that $64 \%$ of health care professionals had seen the disruptive behaviour amongst doctors, whereas the study by Alan H Rosenstein 26 showed that $90 \%$ of the respondents have confirmed to have witnessed disruptive behaviour amongst doctors. In an another study by same author ${ }^{14}$ at a different health care setup showed that $74 \%$ respondents had witnessed the disruptive behaviour amongst physicians. Further breakdown of same data of Alan $\mathrm{H}$. Rosenstein study brought out that $49 \%$ physicians and $86 \%$ nurses said to have witnessed it as compared to present study where $64 \%$ doctors and $66 \%$ nurses had seen disruptive behaviour amongst doctors.

On the question of as to what percentage of doctors exhibit disruptive behaviour, 28 percent said only one percent doctors show disruptive behaviour and another 31percent said 2-3\% percent doctors show disruptive behaviour. Alan H. Rosenstein reported that only 1-3percent physicians are seen with disruptive behaviour ${ }^{14}$. Leape and Fromson documented that only 3 percent to 5 percent of physicians present with a problem of disruptive behaviour. ${ }^{17}$ 
In present study 65 percent of respondents wrote that it is the same doctors who are responsible for disruptive behaviour time and again. Norman T. Reynolds study states that 70 percent of the respondents reported that disruptive behaviours in physician nearly always involved the same physicians. ${ }^{18}$

The values in present study for doctors and nurses witnessing disruptive are in variance however the percentage of doctors exhibiting disruptive behaviour is similar to above study. The reason for variation may be due sampling procedure as the author Alan H. Rosenstein had drawn sample by email as compared to physical sampling in present study and another reason may be variation in tolerance levels of Indian population but it is relevant that only small percentage of doctors (1-3\%) who exhibit disruptive behaviour.

Gender also influences behaviour of doctors ${ }^{19}$. The present study revealed that $57 \%$ respondents reported a greater tendency amongst male doctors, $18 \%$ in female doctors and $25 \%$ did not find any influence of gender. Alan $\mathrm{H}$. Rosenstein ${ }^{14}$ reflects that $57 \%$ respondents reported greater tendency of disruptive behaviour amongst male physicians and only $2 \%$ in female physicians.

On Seeking information regarding frequency of disruptive behaviour amongst doctors, 11\% of health staff observed it on daily basis, 18\% saw it once a week, $29 \%$ once a month and 34\% reported only 1-2 times in a year. The study by Kevin ${ }^{20}$ reported that this was seen daily by $9.5 \%$ whereas $30 \%$ said it happens weekly, $25.6 \%$ monthly and $2.9 \%$ reported once a year

The most frequent type of disruptive behaviour in our study as reported by $56.5 \%$ health care professionals was shouting or yelling, 46.74\%, persons reported not following laid down rules/regulations and protocol, $30.46 \%$ disrespectful interaction, $13.7 \%$ non cooperation, $12.2 \%$ not responding on phone, 8.5\% Insult in front of staff and colleagues, $7.7 \%$ discrimination, $6.9 \%$ throwing instruments or objects, $2.8 \%$ speaking ill of colleagues and $2.4 \%$ respondents reported addiction however cast remarks and slapping was reported as very minimal. Owen MacDonald (21) study quoted 59\% respondents witnessed insults, 54\%, yelling or shouting another $54 \%$ refusing to cooperate with other health care providers, $55 \%$ do not follow established protocols, $14 \%$ substance abuse, 14\%, throwing objects, 3\% physical assault. The frequency of disruptive behaviour parameters noted in this our study is comparatively less except for yelling or shouting which is more. David 0. Weber 22 cited use of condescending language or voice intonation by 88 percent, impatience with questions by 87 percent and reluctance or refusal to answer questions or phone calls by 79 percent., strong verbal abuse by 48 percent, threatening body language by 43 percent and even physical abuse by 4 percent.

There are very few published studies documenting the ill effects of disruptive behaviour on psychological and behavioural variables and the resulting impact on patient care. As mentioned above, research conducted by the IOM, JCAHO and other organizations that promote patient safety have shown a strong correlation between human factors and medical errors and adverse events (23, $24,25)$. Disruptive behaviour by doctors adversely affect the behavioural and psychological responses of staff. In present study $83 \%$ respondents wrote that they felt stressed by disruptive behaviour of doctors, $76 \%$ felt frustrated, $61 \%$ reported loss of concentration for work and $43 \%$ said that they do not feel like communicating with such doctors leading to reduced communicability. $51 \%$ respondents were emphatic in stating that it leads to impairment of relation with such doctors and $47.7 \%$ replied that it leads to reduction in teamwork. Alan H. Rosenstein 14 study shows that $94 \%$ respondents perceived that disruptive behaviour was to responsible for stress and frustration, 
$83 \%$ reported loss of concentration, $90 \%$ respondents quoted reduced cooperation and $91 \%$ respondents said that it leads to reduced communication.

CONCLUSION: No sound and scientific literature of such study is available in Indian scenario. This study was designed to assess the views of doctors, nurses and other health care workers about disruptive or intimidating behaviour shown by doctors in health care setup, its overall impact on behavioural and psychological responses on health care professionals, their morale, job satisfaction and patient care. While most of the respondents reported having witnessed the disruptive behaviour in the hospital setting where they have been working, however respondents viewed this behaviour and its consequences differently. It was brought out that it affects the attitude, efficiency, accuracy, safety, and outcomes of patient care. Intimidating behaviour leads to confrontation and unease among those health care professionals who are required to work closely with these doctors. It is one of the major factor in hospital care setting leading to stress and frustration. Health care professionals feel hesitant to communicate with such doctors for the fear of being insulted which ultimately deflect attention from the patient care. Although only a small percentage of doctors exhibit disruptive behaviour time and again, but it can have a profound overall effect on team dynamics, morale, and patient care and this issue is required to be addressed by hospital management.

\section{REFERENCES:}

1. Michael V. Williams. A Systems approach to disruptive behavior in physicians: A case study. Journal of Medical Licensure and Discipline 2004; 90(4): 18- 23.

2. Federation of State Medical Boards. Report of the special committee on professional conduct and ethics Vol 2004: Page 5.

3. Splete H.Treatment program targets disruptive physicians- Reasons for program referral include anger, performance and compliance issues, sexual misconduct and theft. Ob/Gyn News 2006; 40: 34.

4. Crow SM, Hartman SJ, Nolan TE, Zembo M.A. Prescription for a rogue doctor. Clin Orthop 2003; 411: 334-9.

5. Gallup DG. The disruptive Physician: Myth of reality. Am J Obstet Gynecol 2006; 195: 543-6.

6. Rosenstein A, O'Daniel M. Impact and implications of disruptive behavior in the peri-operative arena. J Am Coll Surg 2006; 203: 96-105.

7. Rosenstein, A., Naylor, B. "Incidence and Impact of Physician and Nurse Disruptive Behaviors in the Emergency Department" The Journal of Emergency Medicine, 2011; 43(1): 1-10.

8. Cangelosi JD et al. Factors related to nurse retention and turnover: an updated study. Health Mark Q 1998; 15(3): 25-43.

9. Parker PA, Kulik JA. Burnout, self- and supervisor-rated job performance, and absenteeism among nurses. J Behav Med 1995; 18(6): 581-99.

10. Revicki DA, May HJ. Organizational characteristics, occupational stress, and mental health in nurses. Behav Med 1989; 15(1): 30-6.

11. Lim VK, Yuen EC. Doctors, patients, and perceived job image: an empirical study of stress and nurses in Singapore. J Behav Med 1998; 21(3): 269-82.

12. Patrick S. Managers shoulder burden of retaining staff. Dallas Bus J 2000 Aug 11. http: //dallas.bizjournals.com/dallas/ stories/2000/08/14. 
13. VHA Research finds Disruptive Behavior Common in Operating Rooms; Behavior Linked to Adverse Events, Medical Errors, and Mortality (2007) Retrieved from: http: //www.surgicenteronline.com/hotnews/67h613463885025.html.

14. Rosenstein, AH and O'Daniel, M: Disruptive behavior and clinical outcomes: Perceptions of nurses and physicians. American Journal of Nursing, 2005: 105(1): 54-64.

15. Gerardi, D: The Emerging Culture of Health Care: Improving End-of-Life Care through Collaboration and Conflict Engagement Among Health Care Professionals. Ohio State Journal on Dispute Resolution, 2007, 23(1): 105-142.

16. Weber, D0: Poll results: Doctors' disruptive behavior disturbs physician leaders. Physician Executive, September/October 2004, 30(5): 6-14.

17. Leape, LL and Fromson, JA: Problem doctors: Is there a system-level solution? Annals of Internal Medicine, 2006, 144: 104-115.

18. Norman T. Reynolds, . Disruptive Physician Behavior: Use and Misuse of the Label. Journal of Medical Regulation vol 98: (1): 11.

19. Gray J. Men and women in the workplace. New York: Harper Collins Publishers; 2004.

20. Kevin. How doctors and nurses can exhibit disruptive behavior.Physician January 6, 2010 http: //www.kevinmd.com/blog/2010/01/doctors-nurses-exhibit-disruptive-behavior.html

21. Owen MacDonald C “Disruptive Physician Behavior" Group Publisher, Quantia MD. May 15, 2011

22. David 0. Weber On Target Managing disruptive physician behavior for Safety's sake disruptive behavior must be tamed The Physician Executive, September• October 2004: 19-20

23. Institute of Medicine, editor. To err is human: building a safer health system. Washington, DC: National Academies Press; 2000. p. 5.

24. U.S. Senate committee on health, education, labor and pensions hearing: Addressing direct care staffing shortages.17May, 2001. Washington, DC: Government Printing Office.

25. Larson E. The impact of physician-nurse interaction on patient care. Holist nurs pract 1999; 13(2): 38-46.

26. Rosenstein $\mathrm{AH}$, et al. Disruptive physician behavior contributes to nursing shortage: study links bad behavior by doctors to nurses leaving the profession. Physician Exec 2002; 28(6): 811.

\section{AUTHORS:}

1. Avtar Singh Bansal

\section{PARTICULARS OF CONTRIBUTORS:}

1. Associate Professor, Department of Community Medicine, Adesh Institute of Medical Sciences and Research, Bathinda, Punjab.

\section{NAME ADDRESS EMAIL ID OF THE} CORRESPONDING AUTHOR:

Dr. Avtar Singh Bansal., Department of Community Medicine, Adesh Institute of Medical Sciences and Research, Bathinda, Punjab, Pin - 151101.

Email- avtarsinghbansal@gmail.com

Date of Submission: 15/12/2013.

Date of Peer Review: 16/12/2013.

Date of Acceptance: 27/12/2013.

Date of Publishing: 10/01/2014 